\title{
Cálculo de carga óptima de explosivos para la consecución y trituración económica de Residuos de Concreto y Demolición (RCD)
}

Calculation of explosives optimal load to achieve the economic trituration of concrete and demolition waste (CDW)

\author{
Raúl Arango $^{a}$; Pedro Panché ${ }^{a}$; Jorge Fonseca ${ }^{a}$, John Rincón ${ }^{a}$ \\ ${ }^{a}$ Escuela de Ingenieros Militares, Colombia
}

Correspondencia: Jorge.fonsecab@esing.edu.co,jfrinconf@gmail.com

Recibido: Mayo 11, 2021. Aceptado: Junio 15, 2021. Publicado: Junio 20, 2021

\section{Resumen}

Hace cuatro años se reglamentó, por medio de la resolución 472 del 28 de febrero de 2017, el uso de materiales reciclados en obras civiles y estructuras de concreto dentro de la ciudad de Bogotá para contrarrestar el uso de agregados de fuentes naturales e incentivar prácticas que promuevan la conservación del medio ambiente. El propósito de este artículo es incentivar el uso de explosivos en demoliciones estudiando las granulometrías generadas a través de la optimización de su factor de carga para ser competitivos en la estructura de costos de la trituración de los residuos de concreto y demolición (RCD). Inicialmente se plantea como la estructura del mercado del agregado de fuentes naturales en la ciudad de Bogotá y su comparación con el uso de RCD (residuos de concreto y demolición) actualmente. Después, por medio de 2 tipos de muestras de concreto de 4000 psi de resistencia se simulan estructuras viga y columna respectivamente al detonar con 4 cargas de dosificaciones distintas de indugel. El mecanismo para predecir las granulometrías teóricas, el tipo de explosivo y la dosificación de cada una de las muestras se hace a través del modelo de fragmentación de Kuz-Ram, el cual es comparado con las granulometrías obtenidas de 8 detonaciones en total. Finalmente, se detona el indugel en cantidades conservadoras manteniendo el control de vibraciones; y a la vez la consecución de tamaño deseado para la eficiencia económica requerida para triturar; obteniéndose un factor de carga del explosivo de $1 \mathrm{~kg} / \mathrm{m}^{3}$ para las vigas y de $1,2 \mathrm{~kg} / \mathrm{m}^{3}$ para las columnas.

Palabras clave: Factor de carga, Residuos de concreto y demolición, Granulometrías, Trituración.

\section{Introducción}

Hasta hace 10 años el uso de agregados de fuentes naturales era el único e indispensable recurso para el desarrollo de infraestructura en la ciudad de Bogotá. Después de la promoción a nivel mundial de la conservación del medio ambiente, los efectos derivados de procesos de minería mal desarrollada, el aumento de los precios de los agregados y el cierre de varios parques mineros, el distrito de Bogotá a través del ministerio del medio ambiente, la secretaria distrital de

\begin{abstract}
Four years ago, resolution 472 of February 28, 2017 urging the use of recycled materials in civil works and concrete structures in all over the city of Bogotá, to counteract the use of aggregates from natural sources and encourage practices that promote the environment conservation. The main goal of this study is to encourage the use of explosives in demolitions by studying the granulometries through the optimization of explosive load factor to be competitive in the cost structure of the crushing of concrete and demolition waste (CDW). Starts with a little research of the market structure of natural sources aggregates in the city and its comparison with the use of CDW (concrete and demolition waste) is currently considered. Then, by means of 2 types of concrete samples of $4000 \mathrm{psi}$ of resistance that simulate beam and column structures respectively, are detonated with 4 different doses of indugel. The mechanism to predict the theoretical granulometry, the type of explosive and the dosage of each of the samples is done through the Kuz-Ram fragmentation model, which is compared with the granulometries obtained from 8 detonations in total. Finally, the indugel is detonated in conservative amounts while maintaining vibration control; and at the same time the achievement of the desired size for the economic efficiency required to crush; obtaining an explosive load factor of $1 \mathrm{~kg} / \mathrm{m} 3$ for the beams and $1.2 \mathrm{~kg} / \mathrm{m} 3$ for the columns.
\end{abstract}

Keywords: Charge factor, Concrete and demolition waste, Granulometries, Crushing.

ambiente y el instituto de desarrollo urbano propone incentivar el uso de agregados de reciclados de demolición y construcción (RCD (residuos de concreto y demolición)). Este articulo propone por medio de la optimización del uso de explosivos obtener RCD (residuos de concreto y demolición) prediciendo una granulometría óptima para que el uso de estos recursos en la construcción sea una alternativa económica y técnica para los constructores de vías y edificaciones dentro de la ciudad. Otra razón, del presente artículo es evaluar el costo de las diferentes etapas de trituración, las cuales van de menor a mayor 
(primaria, secundaria y terciaria) dependiendo del tamaño del material a disminuir; incrementando su costo si el material a alimentar es de un tamaño grande por el correspondiente aumento de número de trituradoras a usar.

Para esto se debe tener en cuenta un tipo y dosificación de explosivo adecuado para evitar proyecciones y vibraciones en las detonaciones y además un tamaño de residuo óptimo para que su posterior trituración sea económicamente viable. Este estudio parte de una investigación de tipo inductiva para conocer el contexto comercial del RCD (residuos de concreto y demolición) en comparación del agregado de fuentes naturales; y de una investigación experimental para obtener el tipo de explosivo, su dosificación y las granulometrías generadas para la optimización del proceso de trituración. La investigación del mercado de los precios de los distintos tipos de agregado (Natural y RCD - residuos de concreto y demolición) se realiza en los sectores del sur, centro y occidente de Bogotá por medio de la consulta y encuesta a distintos transportadores $\mathrm{y}$ escombreras de la ciudad; y por medio de canteras y plantas de trituración ubicadas en Villavicencio, Tolima y poblaciones del Norte, sur y occidente de Cundinamarca. Las detonaciones de las muestras para obtener las granulometrías se realizan en una cantera del municipio de Mosquera, Cundinamarca, en acompañamiento del grupo de manejo de artefactos y explosivos (MARTE) ejército nacional para ser analizadas finalmente, en un laboratorio certificado por el IDU y la unidad de mantenimiento vial.

\section{Método}

Como componente del método, para este estudio se empleó el explosivo Indugel plus [1]. Determinando para este propósito el cálculo de factor de carga para la estimación de la granulometría deseada; eje principal del presente artículo de esta investigación. El factor de carga a calcular es una razón matemática que depende de la cantidad de explosivo a usar (medido en $\mathrm{kg}$ de Indugel) y el volumen del material a ser afectado por la detonación de dicha cantidad particular del explosivo (medido en $\mathrm{m}^{3}$ ) [2].

Posteriormente el material triturado debe cumplir una granulometría, ser inspeccionado a nivel de laboratorio por ensayos para agregados gruesos y finos [3]. Las partículas de agregados gruesos y finos de un material, se evalúa por medio de tamices de abertura cuadrada progresivamente decreciente. Este método también se puede aplicar usando mallas de laboratorio de abertura redonda. Las gráficas obtenidas se muestran sobre un eje de coordenadas X vs Y, en donde se mostrarán el tamaño del tamiz vs porcentaje retenido o pasante en un determinado tamiz [4].

Para la predicción de la cantidad de explosivo a utilizar se usa; la ecuación de tamaño medio de fragmentación del autor
Kuznetsov (1973) y la ecuación de la curva de Rosin Rammler mediante el uso de la ecuación de Kuznetsov [5,6].

Finalmente, por medio de un análisis de regresión de los parámetros anteriormente mencionados se determina la distribución uniforme de cada uno de los tamices mediante el índice de uniformidad.

A continuación, se describe las ecuaciones para predecir el tamaño medio de fragmentación, la proporción del material retenido y el índice de uniformidad.

\subsection{Determinación Tamaño Medio de Fragmentación}

Esta variable es estimada por la ecuación de Kuznetsov, Ec.(1), la cual participa tipo de roca, características físicas y termodinámicas del explosivo y geometría de perforación [5,6].

Con la siguiente Ec. (1) se obtiene el tamaño promedio de la fragmentación deseada en cada voladura:

$$
\underline{X}=A\left(\frac{V_{0}}{Q_{e}}\right)^{0.8} Q e^{\frac{1}{6} \frac{E}{115}^{-\frac{19}{30}}}
$$

Dónde:

Qe = masa del explosivo en kilogramo por taladro a cargar

$\mathrm{E}=$ potencia relativa por peso del explosivo a usar. Los valores están disponibles en la hoja técnica del fabricante.

$\mathrm{Vo}=$ Volumen estimado de roca fragmentada por taladro en metros cúbicos.

$\mathrm{X}=$ Tamaño del fragmento medio que se quiere obtener en $\mathrm{cm}$. $\mathrm{A}=$ factor de roca calculado en base al Índice de Volabilidad

\subsection{Curva de Rosin Rammler}

Se emplea para determinar la descripción razonable de la fragmentación de la roca, Ec. (2); tanto para roca obtenida a través de demoliciones controladas como la triturada.

$$
R=e^{-\left(\frac{X}{X c}\right)^{n}}
$$

Dónde:

$\mathrm{X}=$ el tamaño de la malla,

$\mathrm{Xc}=$ el tamaño característico, $\mathrm{n}=$ índice de uniformidad,

$\mathrm{R}=$ proporción de material retenido en la malla, nos da una descripción razonable de la fragmentación en la voladura de rocas.

\section{3 Índice de Uniformidad}

El índice de uniformidad se calcula mediante la Ec (3):

$$
n=\left(2.2-14 \frac{B}{D^{\prime}}\right)\left[\frac{1+\frac{S}{B}}{2}\right]^{0.5}\left(1-\frac{W}{B}\right)\left(\frac{L}{H}\right)
$$

Dónde: 
$\mathrm{B}=\operatorname{burden}(\mathrm{m})$

$\mathrm{S}=$ espaciamiento $(\mathrm{m})$

$\mathrm{D}^{*}=$ diámetro del taladro $(\mathrm{mm})$

$\mathrm{W}=$ desviación estándar $\mathrm{mm})$, de la precisión de perforación (m)

$\mathrm{L}=$ longitud total de la carga $(\mathrm{m})$

$\mathrm{H}=$ altura del banco $(\mathrm{m})$.

\section{Resultados}

Se prepararon dos configuraciones en concreto de 4000 psi de resistencia; uno de forma de cilindros de $40 \mathrm{~cm}$ de diámetro y de $40 \mathrm{~cm}$ de altura para simular las vigas y otro en forma de cubos de $0,125 \mathrm{~m}^{3}$ para simular las columnas de una edificación, en la Fig.1 se ilustra las configuraciones de concreto.
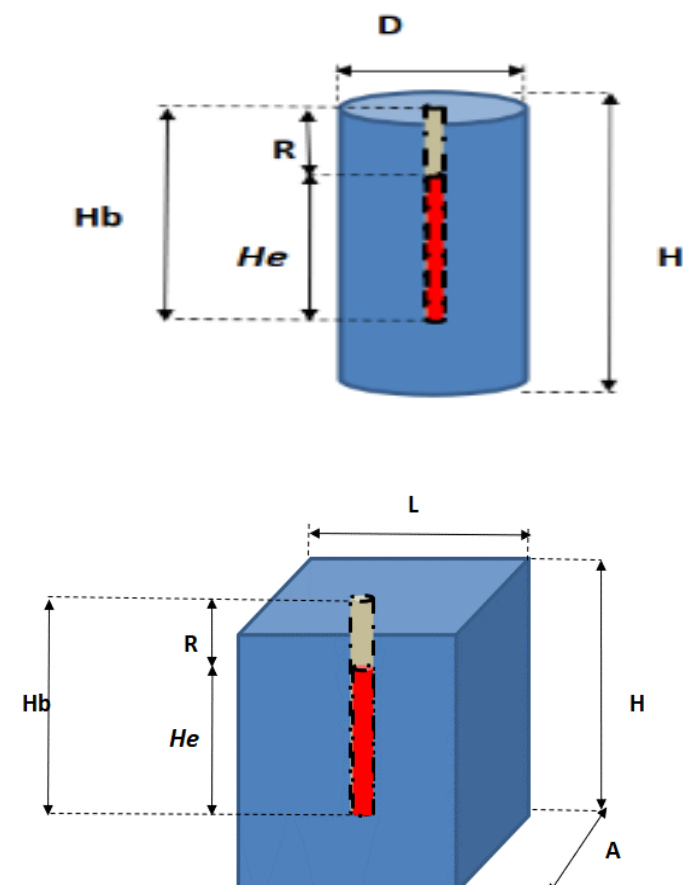

Figura 1. Configuración de concreto cilindro (viga) y (cubo) columna de edificación.

Fuente: Autores.

Para cada configuración, se realizó 4 réplicas adicionando diferentes cantidades de explosivo y evaluar su granulometría a

nivel teórico por medio la modelación matemática de Kuz-Ram; para saber así el diámetro de perforación y la cantidad de Indugel a utilizar. A continuación, se ilustra en las Tab.1 y Tab. 2, las cantidades de indugel para las 4 réplicas de las configuraciones de concreto tanto en cilindros y cubos.
La Tab.1 ilustra las dimensiones de diámetro y longitud de perforación de los barrenos realizados en los cilindros, con la cantidad de retaque y carga explosiva.

Tabla 1. Cantidad de explosivo a utilizar en configuración de concreto forma cilindros (viga) para un volumen $0,0502 \mathrm{~m}^{3}$.

\begin{tabular}{|c|c|c|c|c|}
\hline Tipo de muestra & & $\begin{array}{l}\text { Cilind } \\
\text { (vige }\end{array}$ & & \\
\hline \# & 1 & 2 & 3 & 4 \\
\hline $\begin{array}{c}\text { Indugel }(32 \times 250 \\
\mathrm{mm})\end{array}$ & $\begin{array}{l}1 / 4 \text { barra } \\
\text { Indugel }\end{array}$ & $\begin{array}{l}1 / 2 \text { barra } \\
\text { Indugel }\end{array}$ & $\begin{array}{l}3 / 4 \text { barra } \\
\text { Indugel }\end{array}$ & $\begin{array}{l}1 \text { barra } \\
\text { Indugel }\end{array}$ \\
\hline $\begin{array}{l}\text { Cantidad Indugel } \\
(\mathrm{kg})\end{array}$ & 0,061 & 0,122 & 0,184 & 0,245 \\
\hline $\begin{array}{l}\mathrm{Hb}=\text { Longitud de } \\
\text { perforación }(\mathrm{m})\end{array}$ & 0,094 & 0,190 & 0,280 & 0,380 \\
\hline 0,031 & 0,093 & 0,125 & & \\
\hline
\end{tabular}

La Tab.2 ilustra las dimensiones de diámetro y longitud de perforación de los barrenos realizados en los cubos de concreto, con la cantidad de retaque y carga explosiva.

Tabla 2. Cantidad de explosivo a utilizar en configuración de concreto forma cubo (columna) para un volumen $0,125 \mathrm{~m}^{3}$

\begin{tabular}{ccccc}
\hline Tipo de muestra & \multicolumn{4}{c}{$\begin{array}{c}\text { Cubos } \\
\text { (columnas) }\end{array}$} \\
\hline$\#$ & 1 & 2 & 3 & 4 \\
\hline $\begin{array}{c}\text { Indugel (44x250 } \\
\text { mm) }\end{array}$ & $\begin{array}{c}1 / 4 \text { barra } \\
\text { Indugel }\end{array}$ & $\begin{array}{c}1 / 2 \text { barra } \\
\text { Indugel }\end{array}$ & $\begin{array}{c}3 / 4 \text { barra } \\
\text { Indugel }\end{array}$ & $\begin{array}{c}1 \text { barra } \\
\text { Indugel }\end{array}$ \\
\hline $\begin{array}{c}\text { Cantidad Indugel } \\
(\mathrm{kg})\end{array}$ & 0,116 & 0,232 & 0,348 & 0,464 \\
$\begin{array}{c}\mathrm{Hb}=\text { Longitud de } \\
\text { perforación }(\mathrm{m})\end{array}$ & 0,094 & 0,190 & 0,280 & 0,38 \\
$\begin{array}{c}\text { He= Longitud de } \\
\text { carga (m) }\end{array}$ & 0,062 & 0,125 & 0,187 & 0,25 \\
$\begin{array}{c}\mathrm{R}=\text { Longitud de } \\
\text { retaque }(\mathrm{m})\end{array}$ & 0,031 & 0,062 & 0,093 & 0,125 \\
\hline
\end{tabular}

Las 4 réplicas de cada una de las configuraciones se realizaron perforaciones de diámetro de $32 \mathrm{~mm}$ para los cilindros (vigas) y $44 \mathrm{~mm}$ para los cubos (columnas) como se muestra en las figuras 2 y 3 .

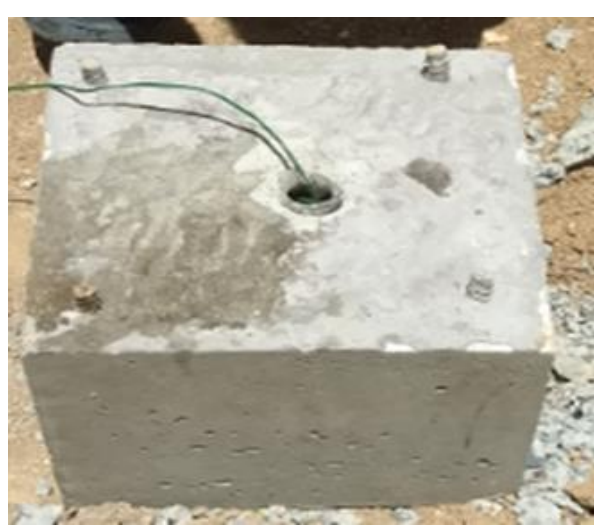

Figura 2. Perforación del cilindro (viga). Fuente: Autores. 


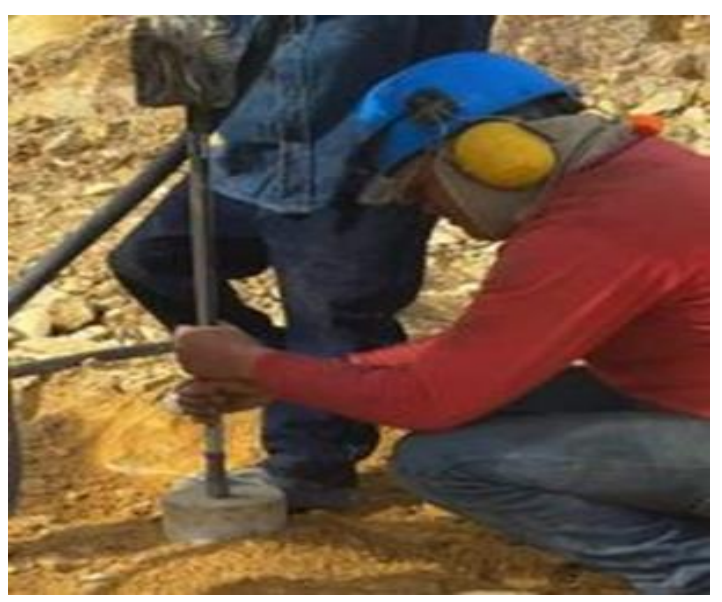

Figura 3. Perforación cubo (columna). Fuente: Autores.

Para las perforaciones se utilizan compresores neumáticos y taladros manuales de perforación de los diámetros requeridos para cada una de 4 réplicas tanto de forma de cilindro o cubos de concreto [6].

Todas las 4 réplicas de cada una de las configuraciones de cilindros y cubos de concreto fueron detonadas; utilizando indugel de 32 × $250 \mathrm{~mm}$ para los cilindros e indugel de 44 × 250 mm para los cubos. Cada replica o muestra se empleó como sistema de iniciación capsula No 8 y mecha lenta, de acuerdo con las Fig. 4 y 5

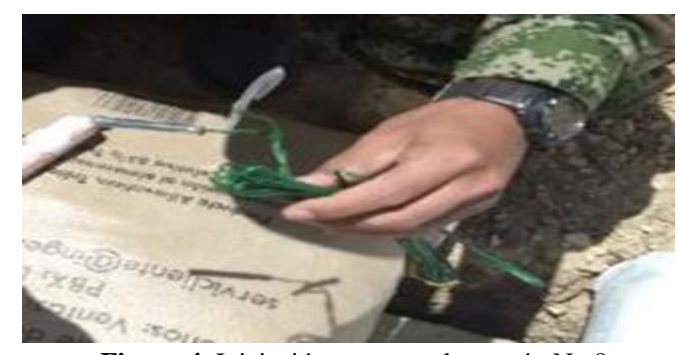

Figura 4. Iniciación con capsula común No.8. Fuente: Autores.

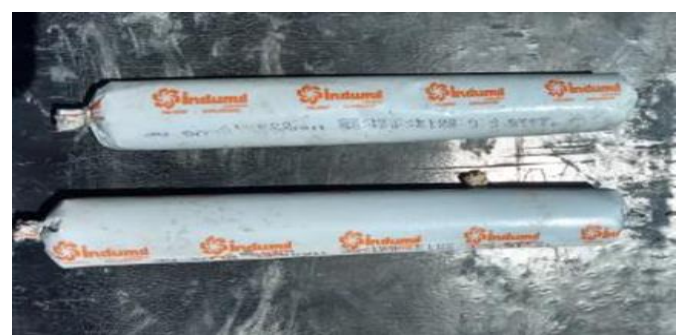

Figura 5. Figura 5. Indugel de 32 x $250 \mathrm{~mm}$. Fuente: Autores.

Las réplicas con su sistema de iniciación fueron enterradas y cubiertas de lonas de caucho en una excavación. Posteriormente detonadas, para así recolectar los residuos de concreto y ser llevados a un laboratorio finalmente para la determinación de su granulometría a nivel experimental.

De otra parte, se estima teóricamente las granulometrías mediante el factor de carga obtenido por medio del modelo de Kuz-Ram para cada una de las configuraciones de concreto. Las gráficas obtenidas y sus resultados se comparan de manera teórica y experimental. Las Fig.6 y 7 que representan los cilindros (vigas) obtenidos tanto teórica y experimentalmente respectivamente.

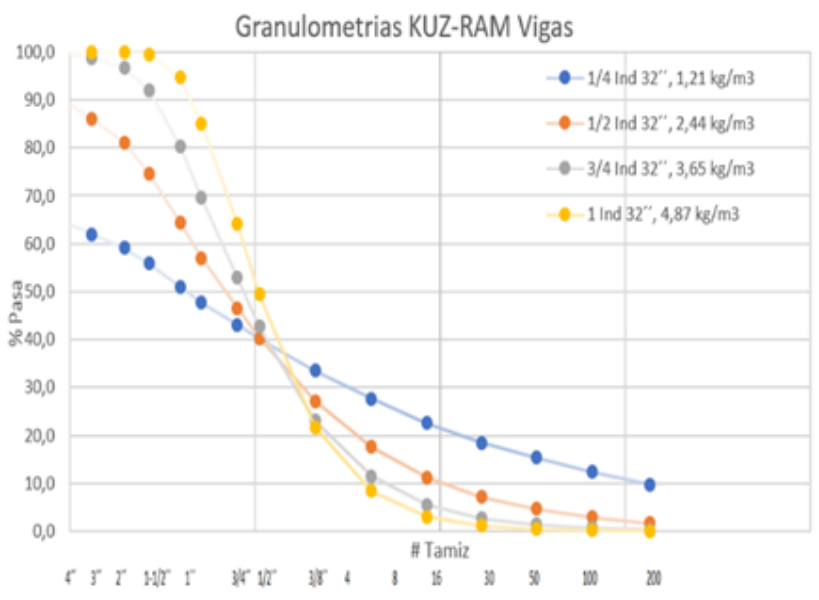

Figura 6. Granulometría teórica y factores de carga Kuz-ram para los cilindros (vigas). Fuente: Autores.

Los resultados de granulometría experimental, Fig.7, obtenidos del laboratorio para cada una de las réplicas se graficó para así comparar con los datos teóricos de la Fig.6 calculados inicialmente y así poder determinar la cantidad de explosivo a utilizar en la práctica para obtención de una determinada granulometría.

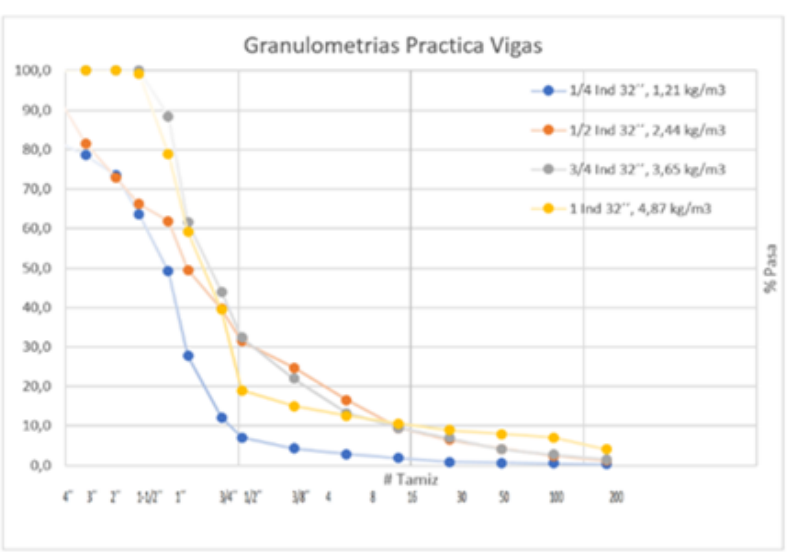

Figura 7. Granulometría experimental y factores de carga para los cilindros (vigas) Fuente: Autores. 
Como se puede apreciar, en la Fig.7, el \% pasante en 4" es cerca del $80 \%$ en las dos primeras curvas (de color naranja y amarillo) con un factor de carga bajo u óptimo. La forma de la curva es parecida al modelo teórico Kuz-ram, Fig.6 y las diferencias es que hay mayor material retenido en la primera muestra y más material pasante a medida que aumenta la carga operante. El tamaño medio de fragmentación esta entre los tamices de 3"y $1,5^{\prime \prime}$, aproximándose a los valores obtenidos por el modelo teórico.

Las Fig. 8 y 9 ilustran los resultados de granulometría a nivel teórico (Kuz-ram) y a nivel experimental para las configuraciones de concreto en forma de cubo, representando

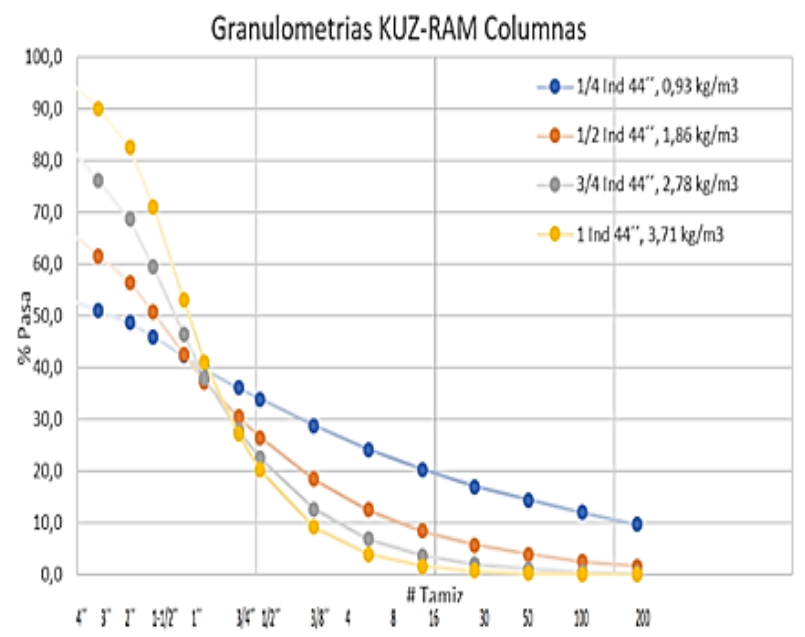

estas las columnas.

Figura 8. Granulometría teórica y factores de carga Kuz-ram para los cubos (columnas) Fuente: Autores.

La fig. 9, ilustra los resultados de granulometría obtenidos experimentalmente para así poder determinar la cantidad de explosivo a utilizar en la práctica para obtención de una determinada granulometría.

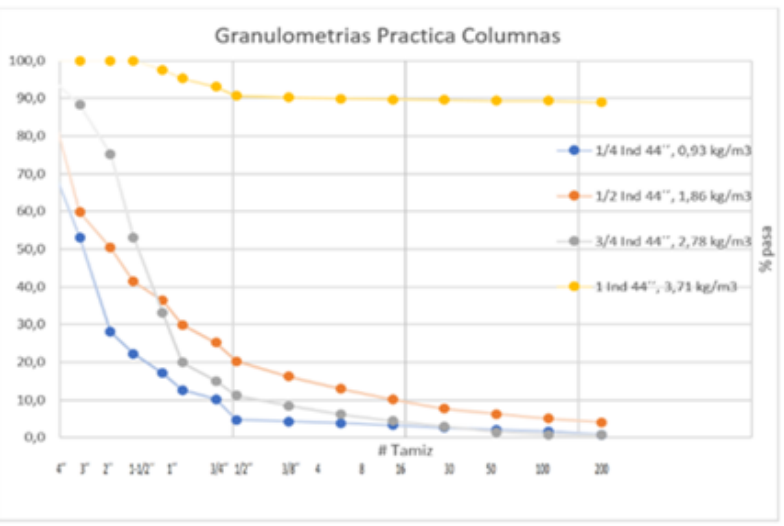

Figura 9. Granulometría experimental y factores de carga para los cubos (columnas). Fuente: Autor(es).
De acuerdo con lo expuesto, aunque las gráficas tienen el mismo comportamiento del modelo teórico a excepción de la réplica 4; pudiendo afirmar que con un factor de carga menor hay menos material pasante y más retenido, contrario a lo que vimos en las gráficas teóricas para los cilindros. El tamaño medio de fragmentación se encuentra entre los tamices 6" y 4". Finalmente se realizan dos cuadros comparativos; Fig.10, de los precios de venta de los RCD actuales provenientes de demoliciones y de los agregados provenientes de fuentes naturales, frente al costo de producción de RCD utilizando Indugel con un factor de carga de $1,1 \mathrm{~kg} / \mathrm{m}^{3}$.

\begin{tabular}{|c|c|c|c|c|c|}
\hline Voladura & $\$$ & 15.700 & Voladura & $\$$ & 15.700 \\
\hline Perforacion & $\$$ & 11.000 & Perforacion & $\$$ & 11.000 \\
\hline Repicado (20\%) & $\$$ & 900 & Repicado (20\%) & $\$$ & 900 \\
\hline \begin{tabular}{|l|} 
Cargue \\
\end{tabular} & $\$$ & 1.500 & Cargue & $\$$ & 1.500 \\
\hline Utilidad & $\$$ & 2.910 & Utilidad & $\$$ & 2.910 \\
\hline Transporte (75\%) & $\$$ & 12.500 & Transporte (75\%) & $\$$ & 12.500 \\
\hline Disposicion (25\%) & $\$$ & 1.850 & Disposicion (25\%) & $\$$ & 1.850 \\
\hline Trituracion & $\$$ & 4.000 & Trituracion & $\$$ & 4.000 \\
\hline Selección & $\$$ & 2.200 & Selección & $\$$ & 2.200 \\
\hline Total & $\$$ & 52.560 & Total & $\$$ & 52.560 \\
\hline Precio de venta & $\$$ & 58.000 & Precio de venta & $\$$ & 82.565 \\
\hline Margen & & $10 \%$ & Margen & & $57 \%$ \\
\hline
\end{tabular}

Figura 10. Costo tentativo $\mathrm{n}$ factor de carga de $1,1 \mathrm{~kg} / \mathrm{m}^{3} \mathrm{y}$ Rentabilidades frente a los actuales productos ofrecidos RCD provenientes de demoliciones manuales (Izquierda) y de agregados de fuente natural (Derecha). Fuente: Autores.

El anterior valor de $1,1 \mathrm{~kg} / \mathrm{m}^{3}$ es obtenido como resultado experimental en el presente artículo de investigación.

\section{Discusión}

La reducción de RCD - residuos de concreto y demolición utilizando Indugel es una opción que permite obtener una granulometría óptima para el aprovechamiento en obras civiles. En la realización de las pruebas se ratificaron los resultados del modelo teórico al acercarse al resultado del tamaño de partícula requerido. Esto se demostró al realizar el tamizado obteniendo diámetros con un tamaño medio de fragmentación entre los tamices de 3" y 1,5 ".

Al realizar la comparación entre los costos de operación obtenidos en la reducción de los residuos de concreto y demolición con Indugel vs los costos de operación en la extracción de agregados en las canteras, se observa, que los costos son iguales. Por otra parte, en el precio de venta se ve una notable diferencia, permitiendo que la utilización del material obtenido de los residuos de concreto y demolición sean presentados como una opción viable para ser aprovechados en obras civiles.

La importancia de reutilizar elementos de demolición para las nuevas obras radica en la cantidad de deshechos que llegan a las escombreras. Estos materiales no eran aprovechados y se 
convertían en un problema ya que en su disposición final terminaban como relleno de humedales.

\section{Conclusiones}

El factor de carga escogido en las vigas fue de $1 \mathrm{~kg} / \mathrm{m}^{3}$ y resulto 2 décimas menor que en las columnas $\left(1,2 \mathrm{~kg} / \mathrm{m}^{3}\right)$, probablemente al mayor volumen de concreto, no obstante, la relación en el comportamiento de su índice de uniformidad y el tamaño medio de fragmentación fue similar en ambas configuraciones, mostrando proporcionalidad e inversamente proporcionalidad respectivamente en ambas geometrías con respecto al aumento del factor de carga

El modelo de KUZ-RAM como factor predictivo de la fragmentación de las voladuras fue útil para indicar qué factores de carga de explosivo a utilizar para alimentar la trituración en aras de economizar el procesamiento de los RCD. Para este caso las curvas obtenidas por los cálculos teóricos tuvieron un comportamiento muy similar de acuerdo con las granulometrías de laboratorio, en cuanto a tamaño medio de fragmentación, factor de uniformidad y en la transición geométrica de las curvas.

De acuerdo a los costos obtenidos y al margen tan grande que hay entre los agregados de RCD y los de fuentes naturales da pie para que haya mayor acercamiento de los proveedores de agregados frente al uso profesional de los explosivos como método más eficiente para obtención de beneficios económico y reducción del impacto ambiental.

\section{Referencias}

[1] Indumil, «Catálogo de Productos,» Industria Militar, Bogotá D.C, 2019.

[2] AEDED,Demolición con explosivos (2018) http://www.aeded.org/informacion/demolicion-con-explosivos.

[3] Secretaría Distrital de Ambiente, Guía para la elaboración del Plan de Gestión de Residuos de Construcción y Demolición - RCD en la obra. (2015)

[4] Universidad del Cauca, Análisis granulométrico de agregados gruesos y finos. (2017)

[5] Konya., Manual de voladura 129. (1975)

[6] Manual de Perforación y Voladura de rocas, 2nd ed, Instituto Tecnológico, y Minero de España (ITGE), Madrid, (2003) 